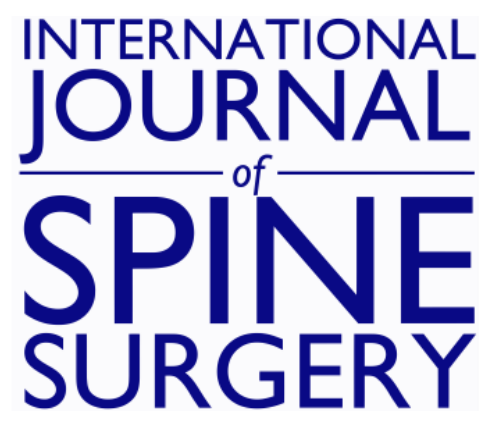

\title{
Prospective Randomized Controlled Trial of The Stabilis Stand Alone Cage (SAC) Versus Bagby and Kuslich (BAK) Implants for Anterior Lumbar Interbody Fusion
}

William Lavelle, Robert F. McLain, Candace Rufo-Smith and David P. Gurd

Int J Spine Surg 2014, 8 ()

doi: https://doi.org/10.14444/1008

http://ijssurgery.com/content/8/8

This information is current as of April 26, 2023.

Email Alerts Receive free email-alerts when new articles cite this article. Sign up at:

http://ijssurgery.com/alerts

The International Journal of Spine Surgery

2397 Waterbury Circle, Suite 1,

Aurora, IL 60504, Phone: +1-630-375-1432 


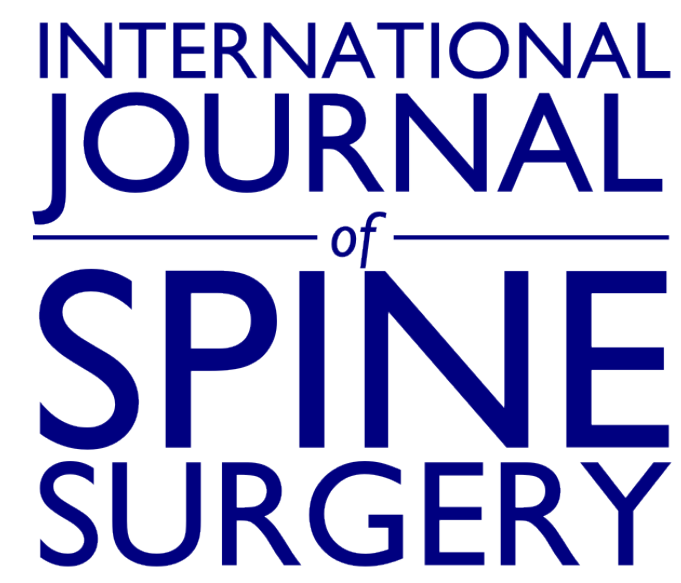

This article generously published free of charge by the International Society for the Advancement of Spine Surgery.

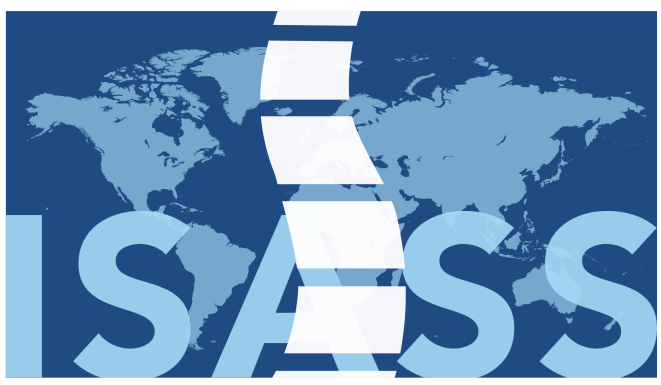

INTERNATIONAL SOCIETY for the ADVANCEMENT of SPINE SURGERY 


\section{Prospective Randomized}

Controlled Trial of The Stabilis Stand Alone Cage (SAC) Versus Bagby and Kuslich (BAK) Implants for Anterior Lumbar Interbody Fusion

William Lavelle, ${ }^{1}$ Robert F. McLain, ${ }^{2}$ Candace Rufo-Smith, ${ }^{2}$ David P. Gurd ${ }^{2}$

${ }^{1}$ SUNY Upstate Medical University, New York; ${ }^{2}$ The Cleveland Clinic, Ohio

\section{Abstract}

\section{Background}

Degenerative disc disease is common and debilitating for many patients. If conservative extensive care fails, anterior lumbar interbody fusion has proven to be an alternative form of surgical management. The Stabilis Stand Alone Cage(SAC) was introduced as a method to obtain stability and fusion. The purpose of this study was to determine whether the Stabilis Stand Alone Cage (SAC) is comparable in safety and efficacy to the Bagby and Kuslich (BAK) device.

\section{Methods}

As part of a prospective, randomized, controlled FDA trial, 73 patients underwent anterior interbody fusion using either the $\mathrm{SAC}(56 \%)$ or the BAK device $(44 \%)$.

\section{Results}

Background characteristics were similar between the two groups. There was no significant difference between the SAC and BAK groups in mean operative time or mean blood loss during surgery. Adverse event rates did not differ between the groups. Assessment of plain radiographs could not confirm solid fusion in $63 \%$ of control and $71 \%$ of study patients. Functional scores from Owestry and SF-36 improved in both groups by the two-year follow-up. There were no significant differences between the SAC and BAK patients with respect to outcome. 


\section{Conclusions}

Both the Stabilis Stand Alone Cage and the BAK Cage provided satisfactory improvement in function and pain relief, despite less than expected radiographic fusion rates. The apparent incongruency between fusion rates and functional outcomes suggests that either radiographs underestimate the true incidence of fusion, or that patients are obtaining good pain relief and improved function despite a lower rate of fusion than previously reported. This was a Level III study.

keywords:

anterior interbody fusion, Bagby and Kuslich Device (BAK), functional improvement, MOS 36 Item Short Form Health Survey (SF-36), Owestry scores, perioperative outcome, radiographic outcome, re-operation, Stabilis Stand Alone Cage (SAC), VAS scores Volume 8 Article 8 doi: $10.14444 / 1008$

\section{Introduction}

Low back pain due to degenerative disc disease continues to be a major public health concern. It remains a leading cause of disability, particularly in the middle-age working population. ${ }^{1}$ Due to the incidence of low back pain and the associated expense of treatment and lost time from work, multiple avenues of treatment have been sought. The mainstay of surgical treatment after exhaustive conservative measures is stabilization, or fusion, of the involved disc space. The primary goal of treatment is to relieve pain and improve patient function. ${ }^{2}$ Fusion has been performed in a multitude of ways either through an anterior, posterior, or combined (360-degree) approach, with or without instrumentation. With a variety of available surgical approaches, stand-alone interbody fusion remains a common procedure.

When assessed against other approaches for lumbar fusion, the anterior interbody technique offers some potential advantages as compared to posterior interbody techniques. Posterior lumbar interbody fusion (PLIF), as well as, transforaminal interbody fusion (TLIF) only allow for the insertion of relatively small cages and bear the risk of collapse and pseudarthrosis unless the surgical technique is meticulous. ${ }^{1,3}$ Pedicle fixation systems may generate serious complications to include screw misplacement.4 The posterior approach may cause paraspinal muscular injury leading to "fusion disease." ${ }^{5}$ McAfee noted a high rate of failure with isolated posterior pedicle screw fixation. ${ }^{6}$ Anterior approaches offer the potential to avoid these complications, however, the risk of injuries to visceral, vascular and genitourinary structures remain a concern.

Biomechanically, two "stand alone" anterior threaded Bagby and Kuslich (BAK) cages result in equivalent stability within the calf spine compared to a PLIF bone graft and pedicle screw construct. ${ }^{7}$ Compared with 360 -degree procedures, anterior interbody fusion(ALIF) has been reported to provide comparable fusion rates with decreased operative time, less blood loss, a shorter hospital stay, less postoperative pain, and no paravertebral muscle necrosis. ${ }^{8}$ 
The hypothesis of this study was that the Stabilis Stand Alone Cage(SAC) would demonstrate comparable efficacy and safety when compared to the BAK cage, the only ALIF device approved by the FDA for stand-alone placement at the time of this trial( December 8, 1998 and December 8, 2002).

\section{Materials and Methods}

This was a randomized prospective FDA trial, designed prior to approval of the Stabilis Stand Alone Cage (SAC). Patients with degenerative lumbar discs and intractable low back pain were enrolled in a randomized, prospective, controlled FDA trial. Indications for the anterior lumbar interbody fusion included: primary diagnosis of degenerative disc disease (discogenic back pain with degeneration of the disc confirmed by medical history and radiographic evaluation) at one or two contiguous levels in patients between 21 and 65 years of age; and chronic, disabling low back pain of at least six months' duration, unresponsive to conservative measures. Exclusion criteria for this study included previous attempts at fusion, active systemic or local infection, severe osteoporosis, immunological suppression, individual was a prisoner, pregnancy, morbid obesity, vascular abnormalities, previous abdominal retroperitoneal operation, or if the patient planned to relocate to another geographic area prior to the completion of the study. Patients younger than 21 years or older than 65 were also excluded from the study.

Patients underwent anterior lumbar interbody fusion for degenerative disc disease of the L4-L5 and/or the L5-S1 levels. Seventy-three patients, from four institutions, were selected for analysis at a minimum of 12 months and a mean of two years follow-up to determine initial outcomes and function and pain profiles at 1 to 4 years. Appropriate Institutional Review Board(IRB) approval was obtained at each participating institution before initiating this study. Patients were randomized into two groups per an FDA approved protocol. Randomization was determined by a central randomization schedule, developed and managed by an independent biostatistician. Once patients had consented for surgery; consented for participation in the study; and enrolled, the surgeon would contact the independent biostatistician to determine the type of interbody fusion system to be utilized. Subjects were not blinded as to which device was implanted. Forty-one patients were randomized to the SAC implant, and thirty-two to the BAK implant.

All patients who met inclusion criteria, with no exclusion criteria, were offered enrollment into the study. Interested patients were provided full informed consent, and signed a patient informed consent document approved by each of the institutions. Indications for the ALIF include primary diagnosis of degenerative disc disease (discogenic back pain with degeneration of the disc confirmed by medical history and radiographic evaluation) at one or two contiguous levels in patients between 21 and 65 years of age; and chronic, disabling low back pain of at least a six month duration, unresponsive to extensive conservative measures. Exclusion criteria for this study included previous attempts at fusion, active systemic or local infection, severe osteoporosis, immunological suppression, incarceration, pregnancy, morbid obesity, vascular abnormalities, previous abdominal retroperitoneal operation, or if the patient planned to relocate to another geographic area prior to the completion of the study. Patients, younger than 21 or older than 65, were also excluded from the study. No unauthorized use of the SAC device was permitted. 
An anterior open approach was used for all cases. The choice of either a transperitoneal or retroperitoneal approach was based upon individual surgeon preference. The transperitoneal approach was typically made through a midline vertical skin incision with fascial split between the rectus muscles. The small bowel was retracted superiorly and the sigmoid colon to the left laterally. The aorta and vena cava usually bifurcate at the level of the L-5 vertebral body. To gain access to the L4-L5 disc space, the aorta and vena cava were retracted to the right after control and ligation of the iliolumbar vein. Access to the L5-S1 disc space was accomplished by entering the retroperitoneum distal to the bifurcation of the vessels. Ligation and control of the middle sacral artery and vein was necessary for complete disc exposure. Dissection over the disc space is performed bluntly, with minimal use of cautery, to avoid injury to the presacral nerve plexus as damage to the plexus may cause retrograde ejaculation in men. ${ }^{5}$

The retroperitoneal approach was typically made through a paramedian incision made from midline to the left, measuring three to four inches. The anterior rectus sheath was divided, and blunt dissection beneath the arcuate line provided entry to the preperitoneal space. Dissection was carried laterally to the left until the psoas muscle could be identified. The peritoneal contents were retracted to expose the retroperitoneal space overlying the spine. The ureter and sympathetic chain were protected at all times. From this point the dissection was similar to the transperitoneal approach. ${ }^{5}$

A small anterior incision and ICBG harvest was carried out for every case, per protocol. Additional reamings from the taps were combined with this. There was no use of Bone Morphogenic Protein (BMP) in any case and allograft bone alone was considered inadequate for use in this trial.

Each patient returned at standard postoperative time periods of 3, 6, 12, and 24 months, and annually thereafter. At each of these time periods, patients were assessed clinically, radiographically, and through standardized patient satisfaction forms. Radiographic examination consisted of anteroposterior, lateral, and flexion/extension views. However, flexion/extension was not performed at the 3-month interval, but was performed at each subsequent visit. An independent radiologist reviewed all of the radiographs. Key determinants of the radiographic assessment include continuous trabecular bridging, radiolucency around the device, migration of the device, and motion of the device. Fusion was defined as presence of bridging trabecular bone between vertebral bodies, no radiolucency greater then $2 \mathrm{~mm}$ in width or surrounding $>50 \%$ of the implant, less than five degrees of angular motion, and less than $3 \mathrm{~mm}$ of translational movement. To be judged as "absolutely fused", the radiographs had to satisfy all of these criteria.

Standardized forms to assess patient satisfaction were completed both preoperatively and postoperatively. The Modified Oswestry Low Back Pain Disability Questionnaire was utilized to quantify the level of back pain. Scores of 0-20 indicate minimal disability; 20-40 indicate moderate disability; 40-60 indicate severe disability; 60-80 indicate crippled; and 80-100 indicate bed-bound or extreme exaggeration. The preoperative score was used as a baseline, and postoperative scores used to subjectively determine changes in pain associated with the procedure. The MOS 36 Item Short form Health Survey (SF-36) was used to acquire a patient-based assessment of general, physical, and mental health, not specifically related to the back. Scores can range from 0-100 with higher scores indicating better function. Scores were followed both preoperatively and 
postoperatively to determine changes associated with the procedure. Pain was assessed on a ten-point visual analogue scale where higher scores relate to worse pain and lower scores relate to less pain. Pain scores were performed both preoperatively and postoperatively at each follow-up visit.

\section{Statistical Methods}

Preoperative, intraoperative, and follow-up examination data were entered into a studyspecific database. These data included details of surgery, fusion status, functional status, and complications. Quantitative data were summarized using means, standard deviations, and percentiles (including median and extreme values). The control and study groups were compared with respect to quantitative variables using the two-sample T-test. Qualitative data were summarized by frequencies and percents. Comparisons with respect to qualitative data were performed with a Chi-squared test, or Fisher's exact test in cases of low event rates. Statistical significance was defined as $\mathrm{p}<0.05$ for all parameters.

\section{Results}

Table 1 shows details of the patient population. Seventy-three patients were enrolled through the authors' respective institutions. The anterior approach was used for all 73 cases, 56 of which were transperitoneal and 17 were retroperitoneal. Nineteen (26\%) of cases involved the L4-L5 disc space, 51\% (37/73) of cases involved the L5-S1 disc space, and $23 \%$ (17/73) of cases involved both the L4-L5 and L5-S1 disc spaces. At the time of data collection for this ongoing study, $89.0 \%$ (65) of patients were assessed at 12-month follow-up and $56.2 \%$ (41) of patients were assessed at 24 months.

Table 1. Demographics

\begin{tabular}{|l|l|l|l|}
\hline & BAK (32) & SAC (41) & p value \\
\hline Age & 45.6 & 43.0 & 0.24 \\
\hline Height (in.) & 67.5 & 67.7 & 0.87 \\
\hline Weight (lbs) & 183.8 & 175.7 & 0.37 \\
\hline Male & 15 & 17 & 0.64 \\
\hline Female & 17 & 24 & 0.64 \\
\hline Substance Dependence & 0 & 1 & 0.99 \\
\hline Current Smoker & 10 & 3 & 0.008 \\
\hline Former Smoker & 9 & 14 & 0.58 \\
\hline Pending Litigation & 1 & 2 & 0.99 \\
\hline Workers Compensation & 8 & 9 & 0.76 \\
\hline
\end{tabular}

Thirty-two patients (44\%) were randomized to the BAK (control group), and 56\% (41/73) of patients were randomized to the SAC (study group). The average patient age was 44.2 years. The average patient height was 67.6 inches and weight was 179.3 pounds (range 115.0 to 285.0$)$. Thirty-two patients (44\%) of the study cohort were male and 56\% (41/ $73)$ were female. Ten patients $(31.3 \%)$ within the control group and three patients $(7.3 \%)$ 
within the study group were current smokers. Nine of the control patients $(28.1 \%)$ and fourteen of the study patients(34.2\%) were former smokers. Pending litigation was a factor for one of the patients in the control group and two of the patients in the study group. Eight patients $(25.0 \%)$ in the control group involved workers compensation versus nine patients $(22.0 \%)$ in the study group. One of patients in the study group had the diagnosis of multiple sclerosis. The groups were assessed for different medical conditions (cancer, cardiovascular, diabetes, immunological, mental disorder, gastrointestinal, hepatic, urogenital, hypertension, metabolic, pulmonary, renal, thyroid, multiple joint disorders) and there were no differences between the groups.

All patients had a primary diagnosis of painful degenerative disc disease unresponsive to nonoperative measures, lasting longer than six months to include exercise based physical therapy, NSAIDs, and pain management which included counseling, medications and injections. Six (18.8\%) of the patients in the control group and ten $(24.4 \%)$ of the patients in the study group had a concomitant disc herniation. Grade 1 spondylolisthesis was present in five (15.6\%) of the control patients and seven (17.1\%) of the study patients. Previous spinal interventions are listed in Table 2. Prior discectomy had been performed in seven $(21.9 \%)$ of control patients and eight $(19.5 \%)$ of the study patients. No prior attempted fusions were performed in either group. The two groups also had equivalent treatment trials with epidural injections, facet joint injections, physical therapy, and medications.

Table 2. Previous Spinal Interventions

\begin{tabular}{|l|l|l|l|}
\hline & BAK (32) & SAC (41) & p value \\
\hline Discectomy & 7 & 8 & 0.80 \\
\hline Epidural Injection & 24 & 30 & 0.86 \\
\hline Facet Joint Injection & 2 & 1 & 0.58 \\
\hline Foramenotomy & 1 & 1 & 0.99 \\
\hline Laminectomy & 8 & 2 & 0.018 \\
\hline Ice/Heat/Hot Bath & 26 & 32 & 0.74 \\
\hline Physical Therapy & 32 & 36 & 0.063 \\
\hline Immobilization & 22 & 28 & 0.97 \\
\hline
\end{tabular}

\section{Perioperative Outcome}

Operative time was slightly less in the study group, but neither time nor estimated blood loss was statistically different between the two groups as shown in Table 3 . The duration of surgery, from positioning to closure, averaged 170.7 minutes (standard deviation 52.5) for the control group, and 160.5 minutes (standard deviation 44.1) for the study group. The total estimated blood loss for the entire group was $184.5 \mathrm{cc}$, ranging from $15 \mathrm{cc}$ to $1200 \mathrm{cc}$. The estimated blood loss for the control group was $182.8 \mathrm{cc}$ (standard deviation 125.0). The study group estimated blood loss was $185.7 \mathrm{cc}$ (standard deviation 194.6). 
Table 3. Operative Information

\begin{tabular}{|l|l|l|l|}
\hline & BAK (32) & SAC (41) & p value \\
\hline Operative Time (min) & 170.7 & 160.5 & 0.37 \\
\hline Estimated Blood Loss (cc) & 182.8 & 185.7 & 0.94 \\
\hline Surgery at L4-L5 & 20 & 17 & 0.075 \\
\hline Surgery at L5-S1 & 20 & 34 & 0.048 \\
\hline Surgery at L4-S1 & 8 & 10 & 0.95 \\
\hline
\end{tabular}

Table 4 lists the complications occurring during the clinical study. There were no deaths, major neurological injuries, or deep infections in the study. One (3\%) control patient and one ( $2 \%)$ study patient had an intraoperative complication. In each case, a small tear of the left common iliac vein was created during the instrumentation. Suture repair of the vessel was accomplished without further sequelae. Retrograde ejaculation occurred in one patient from each group (3\% for control and 2\% for study). Superficial infections of the abdominal and/or the bone graft site occurred in seven patients, four $(10 \%)$ from the study group and three (9\%) from the control group. Each was successfully treated with oral antibiotics.

\begin{tabular}{|c|c|c|c|}
\hline & BAK (32) & SAC (41) & p value \\
\hline Intraoperative Iliac Vein Injury & 1 & 1 & 1 \\
\hline Retrograde Ejaculation & 1 & 1 & 1 \\
\hline Avulsion of Iliac Crest & 1 & 0 & 1 \\
\hline Ventral Wall Hernia & 0 & 1 & 0.44 \\
\hline Reoperation for Adjacent DDD & 2 & 2 & 1 \\
\hline
\end{tabular}

\section{Functional Improvement}

Both groups showed significant functional improvement, although the incremental improvement over each interval was insignificant. For the entire group, the preoperative Oswestry score was 51.84 . This improved to 37.05 by three months and 27.74 by 24 months. Figure 1 graphically illustrates the functional status of patients over time. The preoperative Oswestry score of the control group was 53.6 and improved to 38.6 at the 3 -month interval and 30.4 by 24 months. The preoperative status of the study group was 50.5 and improved to 35.8 by the 3 -month interval and 25.5 by 24 months. Both the improvement seen in the study group and control group reached criteria to be defined as a substantial clinical benefit defined as a 18.8 net improvement, $36.8 \%$ improvement or a final score of $<31.5 .^{9}$ 


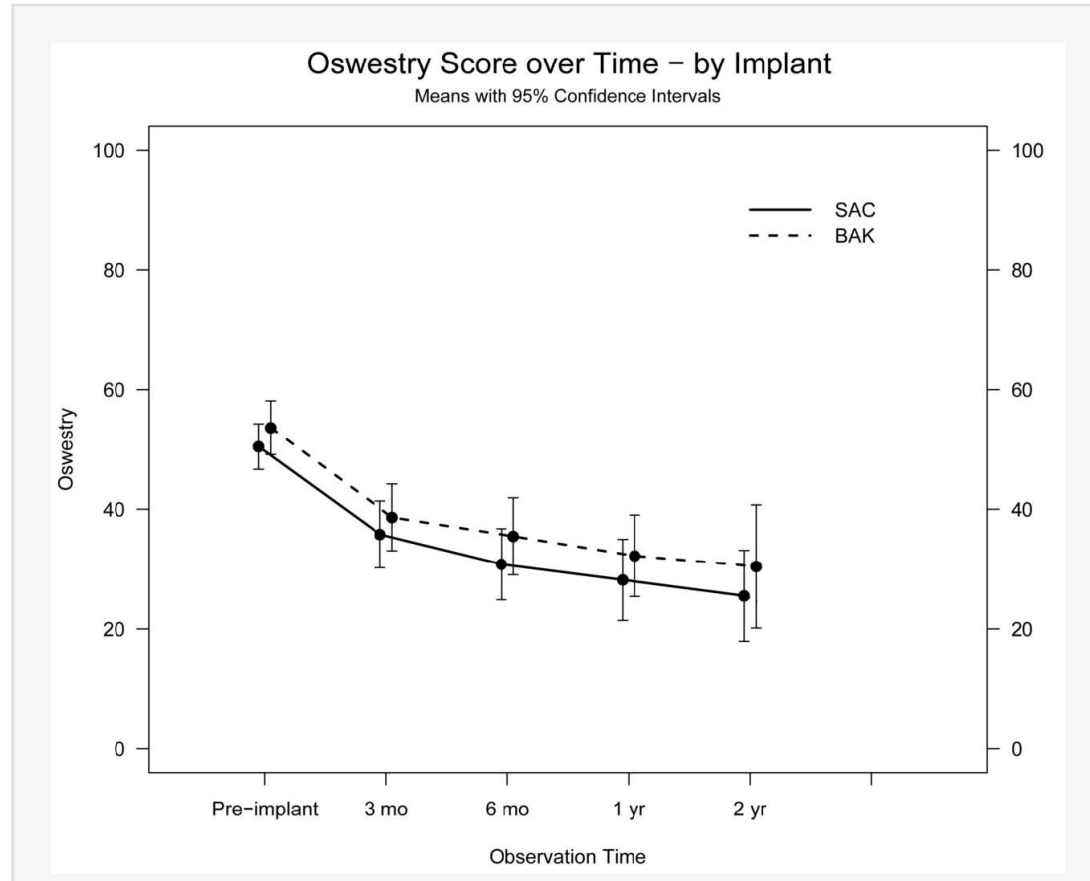

Figure 1. Oswestry Scores are graphically illustrated over time for the study and control population.

The preoperative SF-36 score for the entire group was 7.88. This improved to 18.44 by three months and 46.25 by 24 months. Figure 2 and Figure 3 graphically illustrate the SF-36 results over time. The control group had a preoperative score of 8.6, which improved to 19.2 by three months and 41.7 by 24 months. The study group had a preoperative score of 7.3, which improved to 17.9 by three months and 50.0 by 24 months. This again represented a substantial clinical benefit defined as a 6.2 net improvement, 19.4\% improvement of a final score of $>35.1 .^{9}$ 


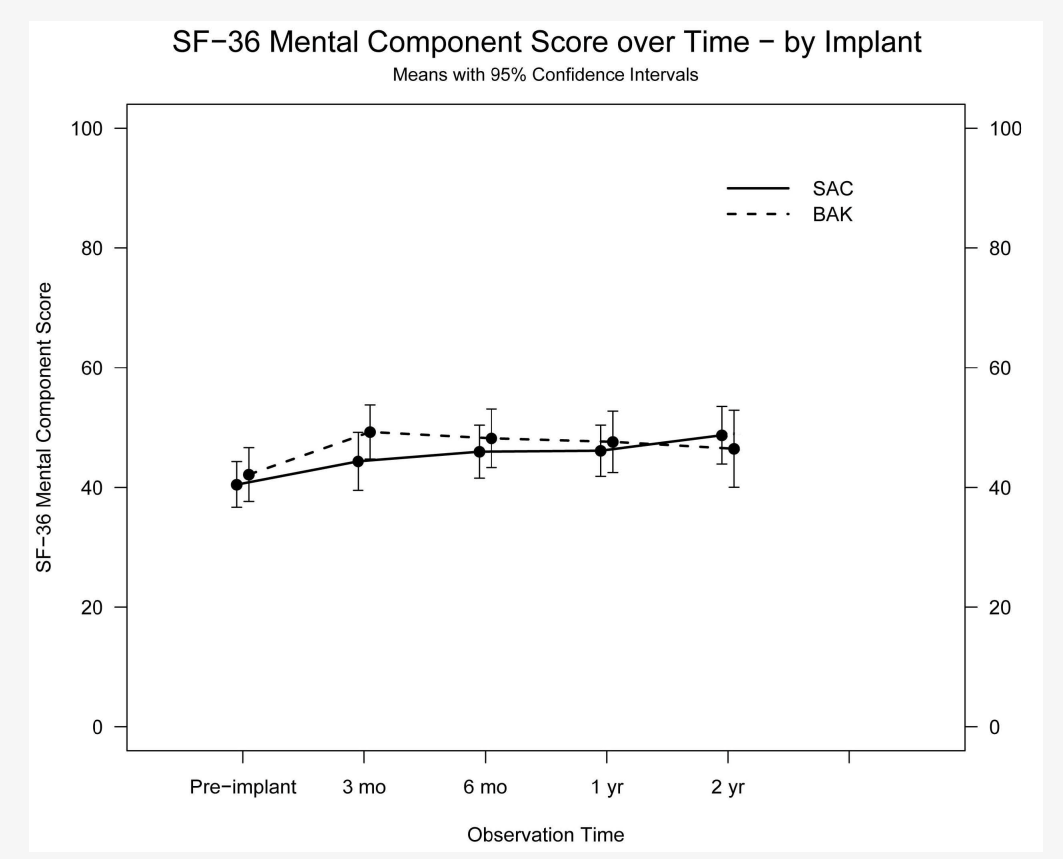

Figure 2. SF-36 Mental Component Scores are graphically depicted over time for the study and control population.

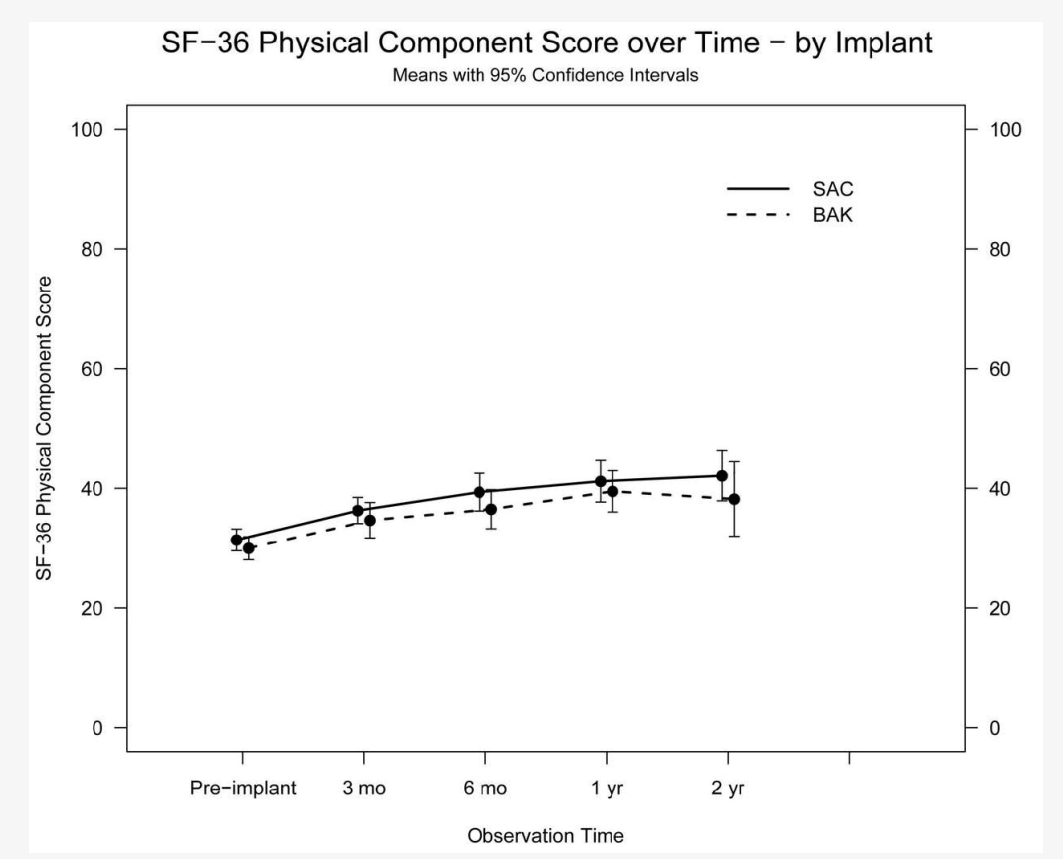

Figure 3. SF-36 Physical Component Scores are graphically depicted over time for the study and control population.

The Oswestry scores showed the most rapid improvement at three months postoperatively. Although perceived functional improvement declined in rate, patients still demonstrated incremental improvement one year after the operation. 


\section{Pain}

Figure 4 depicts back pain score changes over time. Preoperative Visual Analog Scale (VAS) pain scores for the control group and the study group were 7.4 and 6.9 respectively. Three months after the operation both groups had dramatic improvements in pain, 4.7 and 4.5 respectively. Pain scores two years after the operation were 3.7 and 3.6 respectively.

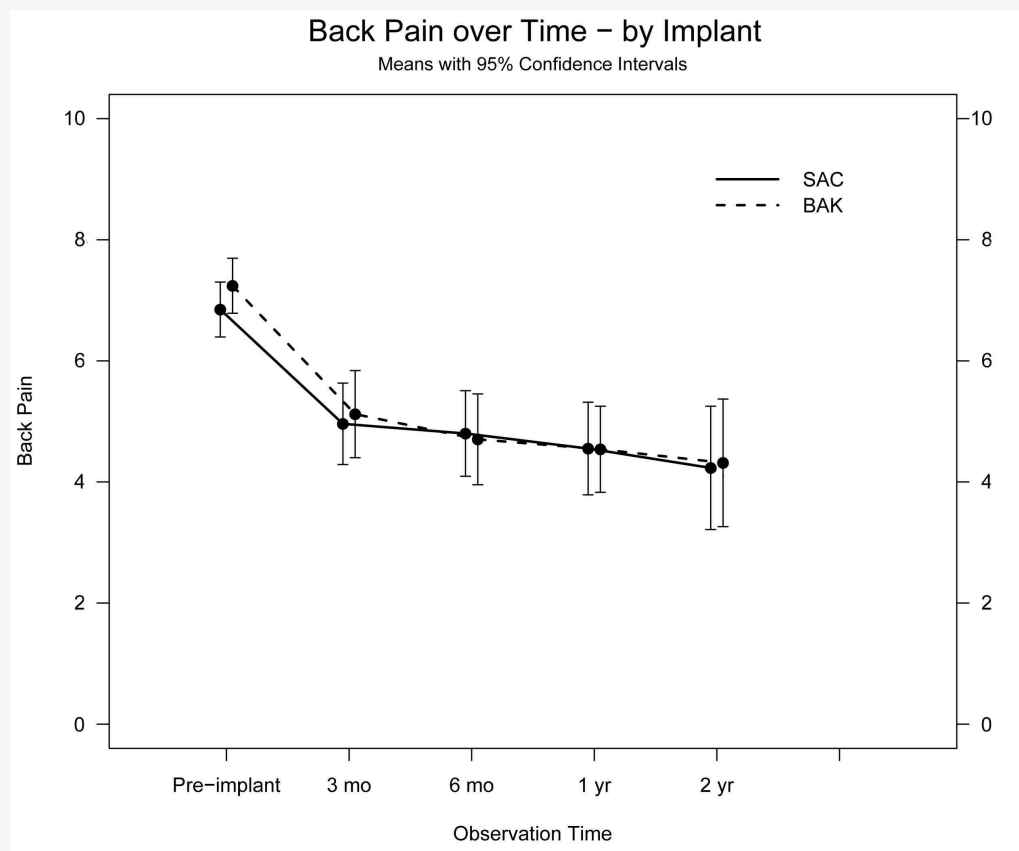

Figure 4. Analog Back Pain Scores are graphically depicted over time for the study and control population.

Lower extremity pain illustrated in Figure 5 and Figure 6 also showed improvement in both groups. Right lower extremity pain for the control group was 4.0 preoperatively and reduced to 2.5 and 1.8 at the three-month and 24-month intervals. Similarly, the study group began at 3.5 preoperatively and reduced to 2.3 and 2.2 at the three-month and 24-month intervals. Left lower extremity pain for the control group was 5.3 preoperatively and reduced to 3.4 and 2.9 at the three-month and 24-month intervals. The study group began at 5.0 and reduced to 3.6 and 2.5 at the three-month and 24-month intervals. There is no statistical difference between the two groups. The pain-score data correlates with the data from the Oswestry data showing the greatest improvement occurred within the first three months. 


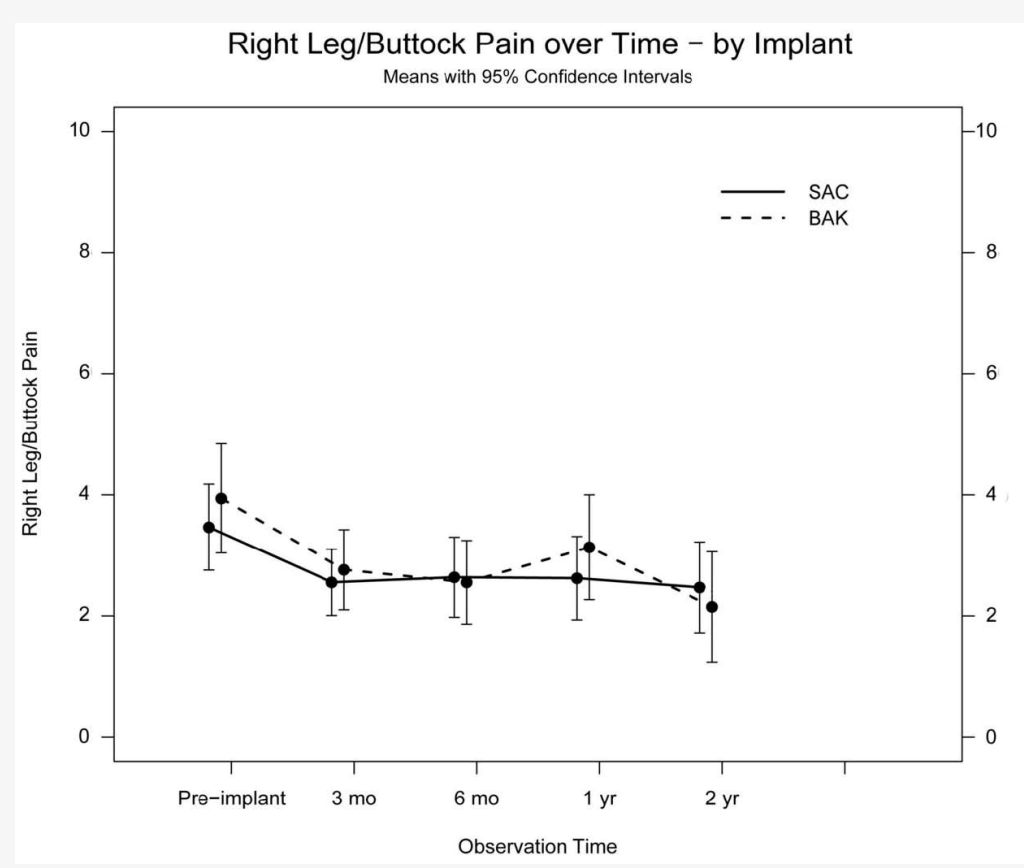

Figure 5. Analog Right Leg Pain Scores are graphically depicted over time for the study and control population.

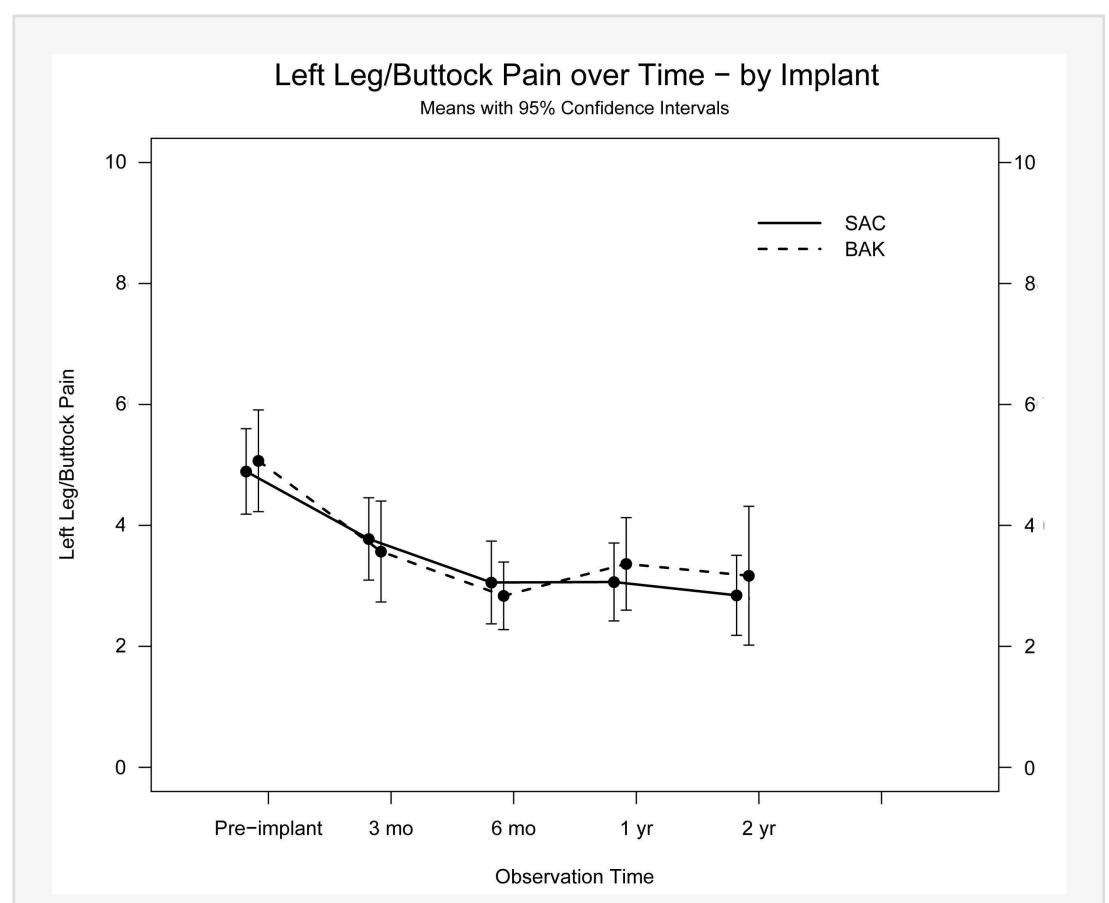

Figure 6. Analog Left Leg Pain Scores are graphically depicted over time for the study and control population.

\section{Radiographic Outcomes}

Radiographs of patients taken two years after the surgery were analyzed by independent radiologists. Data from the flexion and extension images are shown in Figure 7 and Figure 8 . Of the 40 patients who were followed to 24 months, thirteen $(32.5 \%)$ met the 
criteria to be determined as absolute fusion. Seven (36.8\%) of these patients were from the control group and six (28.6\%) were from the study group. There is no statistical difference between the two groups. 


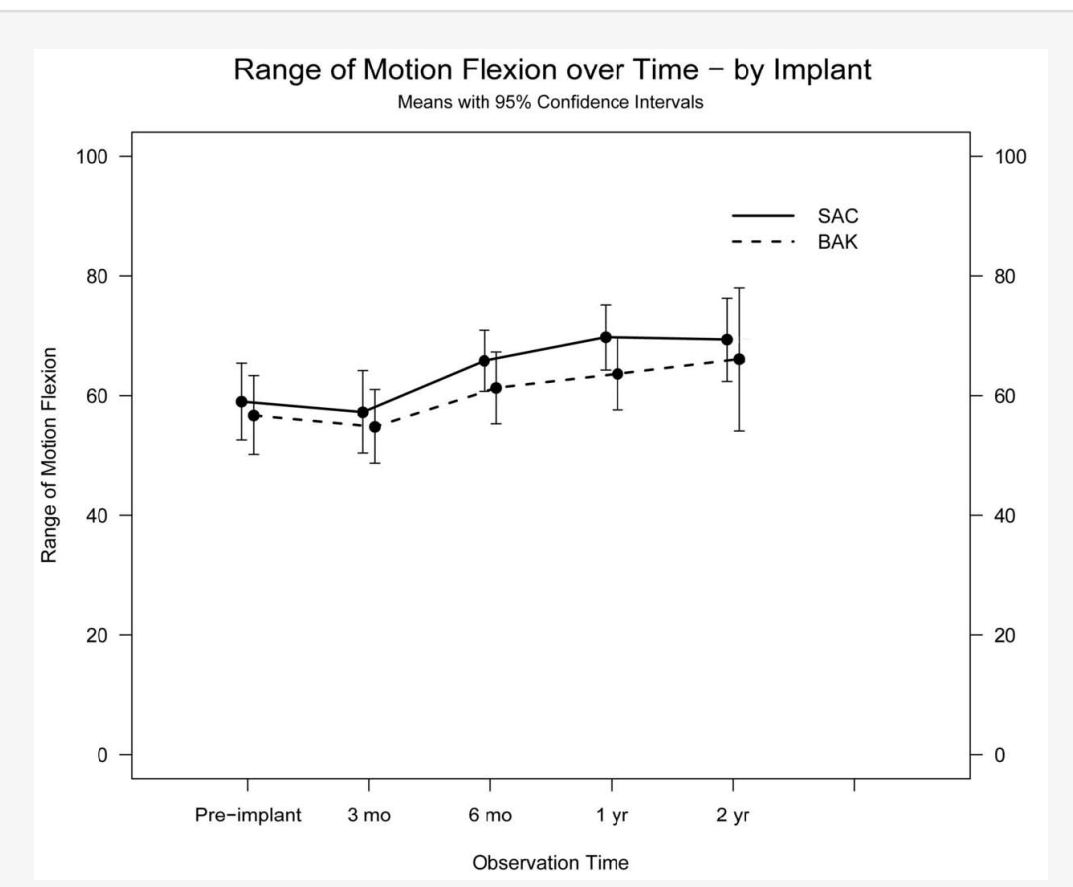

Figure 7. Flexion motion as measured on lateral $\mathrm{X}$-rays over time is shown.

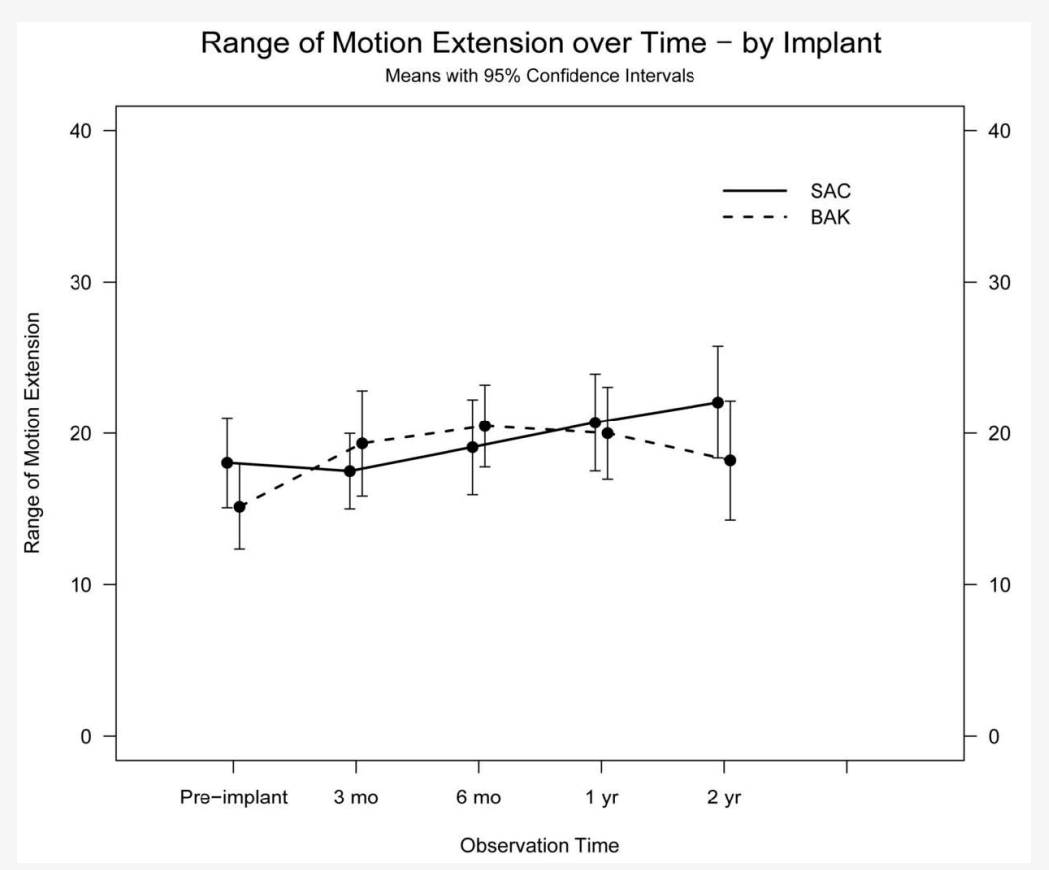

Figure 8. Extension motion as measured on lateral X-rays over time is shown.

The SAC device appeared to better resist subsidence, at least at the L4-L5 disc space. Disc height was measured at each postoperative evaluation and is graphically illustrated in Figure 9 and Figure 10. Two-week radiographs show good distraction of the affected disc space in both groups. The control group L4-L5 disc height was $16.3 \mathrm{~mm}$ and L5-S1 disc height was $19.0 \mathrm{~mm}$, compared to $17.0 \mathrm{~mm}$ and $20.6 \mathrm{~mm}$ respectively for the study 
group. The three-month radiographs show mild changes. At six months, the L4-L5 disc space measures $11.8 \mathrm{~mm}$ for the control group and $17.2 \mathrm{~mm}$ for the study group. The loss of disc height in the control group versus the study group was statistically significant. L5-S1 disc space height decreased to $15.3 \mathrm{~mm}$ for the control group and $17.4 \mathrm{~mm}$ for the study group. By one year, the L4-L5 disc height had decreased $28.8 \%$ for the control group and $1.2 \%$ for the study group. The L5-S1 disc height had decreased $17.4 \%$ for the control group and $20.9 \%$ for the study group. No significant changes occurred after the six-month period. 


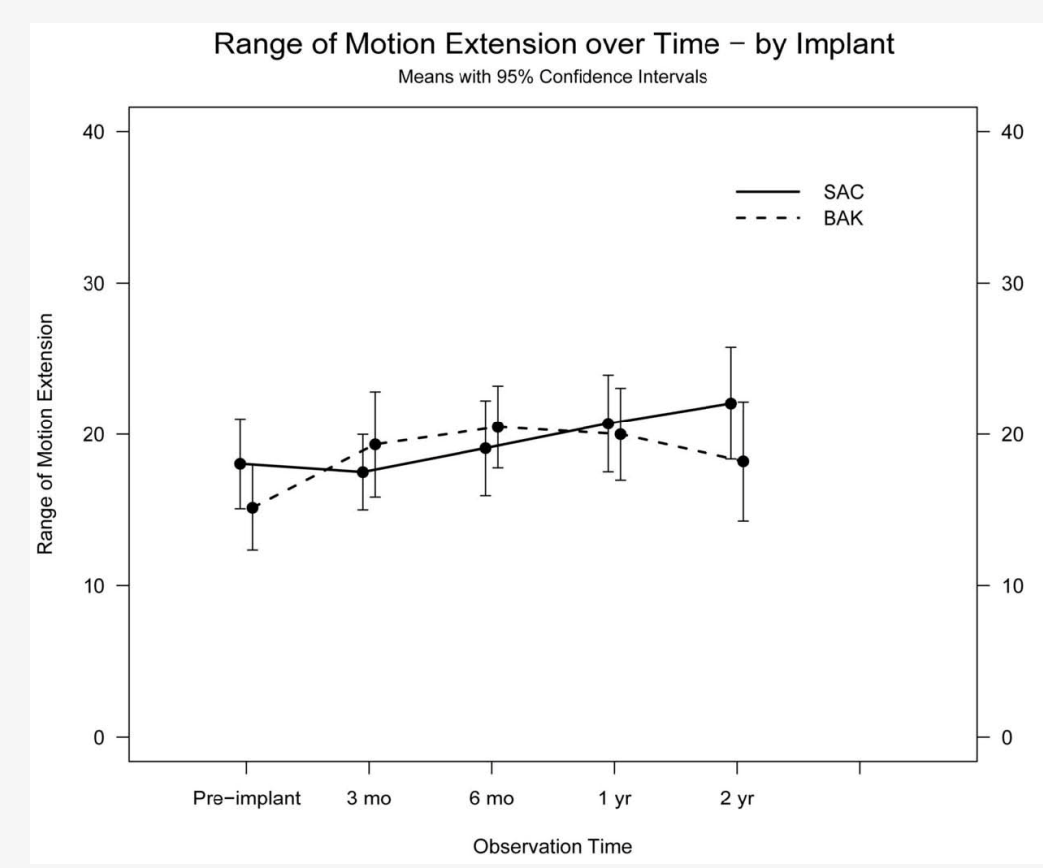

Figure 9. Disc Height at the L4-L5 as measured on lateral X-rays over time is shown.

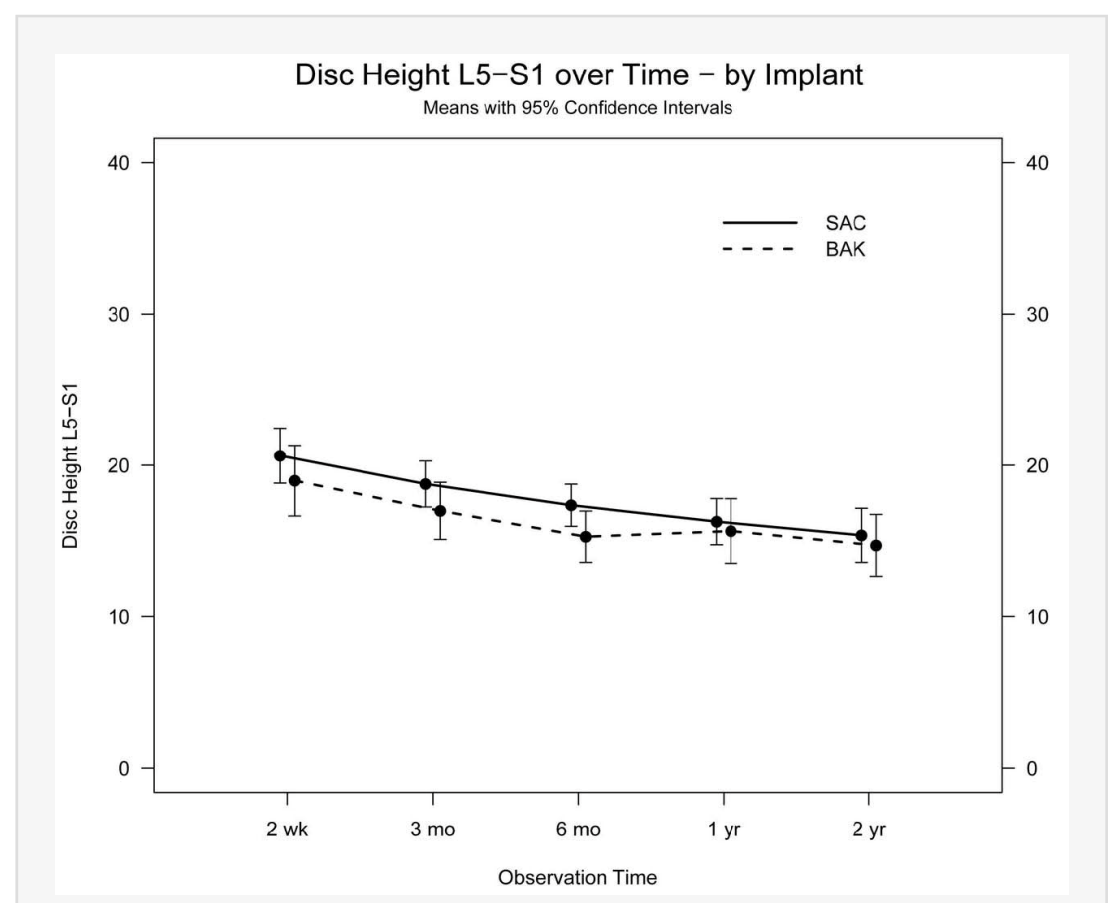

Figure 10. Disc Height at the L5-S1 as measured on lateral X-rays over time is shown.

\section{Re-operation}

Re-operation occurred in nine $(28.1 \%)$ of the control BAK group patients. One patient $(3 \%)$ had an avulsion of the iliac crest at the bone graft site requiring operative fixation. Two (6\%) patients had progression of adjacent level degenerative disc disease. Each had an additional ALIF procedure for the affected level. The remaining six patients (19\%) 
developed a painful pseudarthrosis at the operative level. Each patient was treated with posterior spinal fusion with pedicle fixation. The pseudarthroses all were one level cases, three at L5-S1 and three at L4-L5.

Reoperation occurred in seven (17.1\%) of the study group SAC patients. One patient $(2 \%)$ fell in the early postoperative period sustaining a ventral abdominal wall defect. The patient was taken to the operating room for ventral wall hernia repair. Two patients $(5 \%)$ had progression of adjacent level degenerative disc disease. ALIF was used for one of these patients and the other underwent posterior fusion of the affected level alone. Painful pseudarthrosis requiring posterior spinal fusion occurred in four (10\%) study patients. One of these patients had been treated with a two-level ALIF and the remaining three were L5-S1 cases.

\section{Discussion}

Degenerative disc disease and associated back pain continues to be a major public health concern. As an attempt to address this surgically, multiple devices have been created to assist with anterior lumbar stabilization while osseous fusion occurs. This study assessed two such cages, the SAC and the BAK in terms of their ability to safely and effectively promote fusion as stand-alone devices.

Lumbar interbody fusion is arguably considered the optimal spinal fusion from a mechanical standpoint, because the fusion is directed at the weight-bearing portion of the spine. ${ }^{10}$ With anterior lumbar interbody fusion, there is better blood supply from the endplates to promote an environment conducive to bone formation. ${ }^{3,6,11,12,13}$ Other advantages include optimal weight-bearing (compressive forces) to promote fusion, increase of foraminal height, anterior load sharing, need for less bone graft, distraction of disc height to improved sagittal balance, and prevent risk of flat back syndrome. ${ }^{2,3,6,10,11 \text {, }}$ ${ }^{12,13,14,15}$ Complications include retrograde ejaculation (1.9\%), damage to major vessel (1.7\%), urological (1.4\%), and postoperative ileus $(2.2 \%)$.

The use of bone graft alone has led to respectable fusion rates, but at the risk of subsidence of the affected disc space. Dennis et al. found loss of disc space height in $100 \%$ of patients with allograft and autograft bone alone. ${ }^{18}$ Subsidence occurred in $85 \%$ of cases with femoral strut graft alone in a study by Kumar, et al. ${ }^{19}$ Loss of disc height also causes decreased foraminal size and changes overall spine mechanics. ${ }^{2}$ This has led to the development of structurally sound devices to maintain the disc height until a bony fusion can develop. Bagby did early work with augmentation of the fusion site. ${ }^{20}$ Unacceptable morbidity was found when using iliac crest bone graft alone for spine fusion in horses with Wobbler syndrome. The creation of the first interbody stainless-steel basket, the Bagby basket for thoroughbred racehorses, led to maintenance of the disc height and improved neurological function. Some of these horses not only survived for many years, but also won races.

At the time of this study, the most extensively used and studied interbody cage device was the Bagby and Kuslich Cage (BAK). ${ }^{8}$ In a prospective, four-year follow-up study of 196 patients (122 anterior), $95.1 \%$ of patients had radiographic fusion and $62.7 \%$ were functional enough to return to work. ${ }^{1} 3.1 \%$ of patients required a reoperation for 
pseudarthrosis, 5 of the 6 were after an anterior, 2-level procedure. At 2 years, fusion rates were $93.0 \%$ for ALIF procedures and $90.6 \%$ for PLIF procedures. ${ }^{4} 90.7 \%$ of patients had improved functional status at 24 months.

The demographic background for these two treatment groups was similar. However, there were some potentially significant differences. The control group contained more current smokers (31\% vs. $7 \%$ ) which may have led to an increased risk for pseudarthrosis. Factors such as workers compensation and pending litigation were comparable between the groups. Preoperative diagnosis, as well as prior treatment, was similar between the groups except for previous laminectomy, which was more common in the control group. Inherently, this may add an instability factor to the control group that may lead to pseudarthrosis.

Intraoperative characteristics of the two groups did not differ significantly. The study group required approximately 10 minutes less operative time which is neither statistically or clinically significant. Blood loss and intraoperative complications were similar between the two groups.

Both study groups showed improvement in functional outcome scores. The Oswestry scores decreased by nearly half for each group by the 24-month post surgery mark. This is correlated by a significant increase in SF-36 scores for each group at 2 years. Pain scores demonstrated similar improvement. Each of these subjective factors showed similar improvements that did not differ between the control or study group and reached levels that would be considered clinically meaningful. ${ }^{9}$ Oswestry and VAS scores showed their greatest improvement over the first 3-month interval.

Readers should be cognizant that this study merely compared these two anterior interbody devices. By design, the study did not include a non-operative control group; therefore, no direct conclusions about the efficacy of either device as compared to non-operative treatment can be made based on the study.

Fusion has been a difficult factor to judge. At the time of the initiation of this study, the five-degree of motion plain $\mathrm{x}$-ray radiographic criteria was widely accepted and was designated in the FDA protocol as indicative of fusion in these patients. A different criterion to include a smaller range of motion of the requirement for a post operative CT scan can certainly be supported, but we cannot change the current study design in retrospect. Consensus of opinion states ${ }^{8}$ fusion is likely when there is absence of periimplant lucency, absence of motion on flexion-extension radiographs, and evidence of continuous bony bridging between the vertebrae. It has often been stated that the only way to absolutely confirm the presence of fusion is surgical exploration. Most agree that less than five degrees of motion at the disc space is reasonably suggestive of fusion. Due to the difficulty in determining and defining fusion, it is difficult to compare one study against another for fusion status unless the same criteria are used in each.

This study used a very strict definition of fusion as presence of bridging trabeculae between the vertebral bodies, no radiolucency greater than $2 \mathrm{~mm}$ in width or surrounding $>50 \%$ of the implant, less than 5 degrees of angular motion, and less than $3 \mathrm{~mm}$ of translational movement as judged by an independent radiologist blinded to any other clinical factors. While many subjects met the final three criteria, the difficulty in 
visualizing bridging bone through the mass of the metal implants rendered many of the fusions probable instead of absolute. With these criteria, overall fusion rate was low compared to previous reports of lumbar spine fusion in the literature. Fusion rates may range from $16 \%$ to $95 \%,{ }^{21,} 22$ but most series focusing on the BAK cage report fusion rates of $90 \%$ to $100 \%$, even with the use of only one cage. ${ }^{1,4,11,23,24}$ Our fusion rate results were significantly lower than those reported. This may be due to the criteria used for defining fusion in this study were too stringent. Also, our films were independently reviewed by radiologists who strictly adhered to the defined fusion criteria without accounting for any clinical factors such as the presence or absence of pain, which may influence a clinician's opinion of the fusion status. Another possibility was that it is more difficult to visualize bridging trabecular bone due to implant overlap, or potentially because the bridging bone is traversing within the implant. Still, previous studies have used the BAK device and reported high fusion rates. Regardless of reasoning, the difference in fusion rate between the two groups was not statistically significant, and symptomatic improvement surprisingly exceeded what would be expected for our stated fusion rate. The apparent incongruency between fusion rates and functional outcomes suggest that either radiographs underestimate the true incidence of fusion in these cases, or that patients are obtaining good pain relief and improved function despite a lower rate of fusion than previously reported.

One of the main functions of the ALIF implant is to maintain disc height while awaiting fusion. The benefit of implant usage versus simple bone grafting is the ability to distract and maintain disc height. 2, 3, 6, 10,11,12, 13, 14, 15, 18, 19, 20, 25, 26 Both groups had approximately $20 \%$ loss of disc height at the L5-S1 disc space determined from the postoperative measurements. The L4-L5 disc space was preserved in the SAC group with only a $1 \%$ loss during the first year; while the BAK group lost $28.8 \%$ of the disc height. Maintenance of disc height is very important at these levels. L4-S1 accounts for $67 \%$ of the lumbar spine lordotic angle. ${ }^{15}$ In the article by Goldstein, et al., ${ }^{15}$

they noticed no loss of lordosis when doing an anterior fusion, but a decrease of $3.6 \%$ in the posterior fusion. In a sheep model with the ALIF procedure, there was no loss of disc height after the second month. ${ }^{10}$ Beulter and Peppelman ${ }^{2}$ assessed two types of BAK cages and found only $10 \%$ subsided greater than $2 \mathrm{~mm}$. The fact that SAC shows such improved mechanical stability at L4-L5 over L5-S1 is not currently understood and may be a reflection of a limited volume of patients. Overall, there was settling of the disc space and loss of disc height that seemed greater than the reported incidence in the literature.

The safety of the SAC cage is comparable to the BAK cage. There were no deaths or deep infections in either group. There were no neurological injuries in either group. One intraoperative complication occurred in each group, a tear of the left common iliac vein. This is consistent with the reported $1.0 \%$ incidence of vascular injury within the literature. ${ }^{16}$ The incidence of retrograde ejaculation, one subject in each group, is also consistent with the $0.42 \%$ to $5.9 \%$ incidence reported in the literature. ${ }^{16,17}$

The overall reoperation rate for pseudarthrosis was $13.7 \%$ with no statistical difference between the two groups. Again, the results of this study were not as favorable as previous studies. In the assessment of the BAK cage by Kuslich, et al., ${ }^{1}$ the reoperation rate for pseudarthrosis was $3.1 \%$. McAfee $^{6}$ reviewed the literature for fusion cages and found a reoperation rate of $4 \%$. Other studies find an incidence of reoperation to be as low as 
$2.7 \% .{ }^{16}$ and as high as $22 \% .{ }^{27}$ One possible explanation is that utilizing such a strict and independent radiographic criteria to assess fusion may have influenced surgeons to address continued pain in a patient classified as a pseudarthrosis through a revision surgery that would have otherwise been dismissed as a clinical failure and neglected.

A limitation to this study was the decline in follow-up at two years. One of the goals of the study was to compare surgical and perioperative outcomes of the two devices for safety and surgical complications. Follow-up in all 73 patients was complete as per protocol. Follow-up of a minimum of one year was desired with respect to post-operative complications and fusion, and this was attained in roughly $90 \%$ of patients. Drop-out after that point was marked by patients who chose not to return to regional centers far from their hometown. While phone follow-up determined that these patients were maintaining their outcome results without deterioration, the criteria for inclusion in the study was physical return to evaluation. Hence fall-off was seen at the 24 month period.

\section{Conclusion}

Both the Stabilis Stand Alone Cage (SAC), as well as, the BAK Cage provided satisfactory improvement in function and pain relief, despite having a less than expected radiographic fusion rate. Functional improvements with both groups were comparable at 2-year follow-up to the best results reported for disc athroplasty. The use of an anterior cage may not guarantee maintenance of the disc height.

\section{References}

1. Kuslich SD, Danielson G, Dowdle JD, Sherman J, Fredrickson B, Yuan H, et al. Four-year follow-up results of lumbar spine arthrodesis using the Bagby and Kuslich lumbar fusion cage. Spine. 2000;25:2656-2662.

2. Beutler WJ, Peppelman WC, Jr. Anterior lumbar fusion with paired BAK standard and paired BAK Proximity cages: subsidence incidence, subsidence factors, and clinical outcome. Spine J. 2003;3(4):289-293.

3. Zdeblick TA, Phillips FM: Interbody cage devices. Spine. 2003:28(15 Suppl):S2-S7.

4. Kuslich SD, Ulstrom CL, Griffith SL, Ahern JW, Dowdle JD. The Bagby and Kuslich method of lumbar interbody fusion: History, techniques, and 2-year followup results of a United States prospective, multicenter trial. Spine. 1998;23(11):1267-1278; discussion1279.

5. Zdeblick TA. Anterior lumbar interbody fusion. In: Chapman's, 3rd ed. Orthopaedic Surgery. Philadelphia: Lippincott Williams \& Wilkins; 2001:3809-3816.

6. McAfee PC. Interbody fusion cages in reconstructive operations on the spine. J Bone Joint Surg Am. 1999;81(6):859-880.

7. Brodke DS, Dick JC, Kunz DN, McCabe R, Zdeblick TA. Posterior lumbar interbody fusion: A biomechanical comparison, including a new threaded cage. Spine. 1997;22(1):26-31.

8. McAfee PC, Boden SD, Brantington JW, Fraser RD, Kuslich SD, Oxland TR, et al. Symposium: A critical discrepancy - a criteria of successful arthrodesis following interbody spinal fusions. Spine. 2001;26(3):320-334. 
9. Glassman SD, Copav AG, Berven SH, Polly DW, Surbach BR, Carreon LY. Defining substantial clinical benefit following lumbar spine arthodesis. J Bone Joint Surg Am. 2008; 90(9):1839-1847.

10. Sandhu HS, Turner S, Kabo JM, Kanim LE, Liu D, Nourparvar A, et al. Distractive properties of a threaded interbody fusion device: An in vivo model. Spine. 1996;21(10):1201-1210.

11. McAfee PC, Lee GA, Fedder IL, Cunningham BW. Anterior BAK instrumentation and fusion: complete versus partial discectomy. Clin Orthop Relat Res. 2002;394:55-63.

12. Nord RM, Sandhu HS, Khan SN, Diwan A. Threaded cortical bone dowels in lumbosacral arthrodesis: a review. Clin Orthop Relat Res. 2003;414:101-111.

13. Weiner BK, Fraser RD. Lumbar interbody cages. Spine. 1998;23(5):634-640.

14. Duggal N, Mendiondo I, Pares HR, Jhawar BS, Das K, Kenny KJ, et al. Anterior lumbar interbody fusion for treatment of failed back surgery syndrome: an outcome analysis. Neurosurgery. 2004;54(3):636-643; discussion 643-644.

15. Goldstein JA, Macenski MJ, Griffith SL, McAfee PC. Lumbar sagittal alignment with a threaded interbody cage. Spine. 2001;26(10):1137-1142.

16. Carl AL, Kostuick JP, Abitbol JJ, et al: Interdiscal cage complications: a general consensus. Presented at the Annual Meeting of the North American Spine Society. Chicago Illinois, 1999.

17. Sasso RC, Kenneth Burkus J, LeHuec JC. Retrograde ejaculation after anterior lumbar interbody fusion: transperitoneal versus retroperitoneal exposure. Spine. 2003;28(10):1023-1026.

18. Dennis S, Watkins R, Landaker S, Dillin W, Springer D. Comparison of disc space heights after anterior lumbar interbody fusion. Spine. 1989;14(8):876-878.

19. Kumar A, Kozak JA, Doherty BJ, Dickson JH. Interspace distraction and graft subsidence after anterior lumbar fusion with femoral strut allograft. Spine. 1993;18(16):2393-2400.

20. Bagby, GW: Arthrodesis by the distraction-compression method using a stainless steel implant. Orthopedics. 1988;11(6):931-934.

21. Penta M, Fraser RD. Anterior lumbar interbody fusion: a minimum 10-year followup. Spine. 1997;22(20):2429-2434.

22. Stauffer RN, Coventry MB. Anterior interbody lumbar spine fusion. Analysis of Mayo Clinic series. J Bone Joint Surg Am. 1972;54(4):756-768.

23. Oxland TR, Hoffer Z, Nydegger TD, Rathonyi GC, Nolte LP. A comparative biomechanical investigation of anterior lumbar interbody cages: central and bilateral approaches. J Bone Joint Surg Am. 2000; 82(3):383-393

24. Zhao J, Wang X, Hou T, He S. One versus two BAK fusion cages in posterior lumbar interbody fusion to L4-L5 degenerative spondylolisthesis: a randomized, controlled prospective study in 25 patients with minimum two-year follow-up. Spine. 2002;27(24):2753-2757.

25. Hollowell JP, Vollmer DG, Wilson CR, Pintar FA, Yoganandan N. Biomechanical analysis of thoracolumbar interbody constructs: how important is the endplate? Spine. 1996;21(9):1032-1036.

26. Patwardhan AG, Carandang G, Ghanayem AJ, Havey RM, Cunningham B, Voronov LI, et al. Compressive preload improves the stability of anterior interbody fusion cage constructs. JBone Joint Surg A. 2003; 85-A(9):1749-1756. 
27. Button G, Gupta M, Barrett C, Cammack P, Benson D. Three- to six-year follow-up of stand-alone BAK cages implanted by a single surgeon. Spine J. 2005;5(2):155-160.

\section{Disclosures}

The authors declare no relevant financial disclosures.

Copyright (C) 2014 ISASS - International Society for the Advancement of Spine Surgery. To see more or order reprints or permissions, see http://ijssurgery.com. 